Research Article

\title{
Electroacupuncture Inhibits Atherosclerosis through Regulating Intestinal Flora and Host Metabolites in Rabbit
}

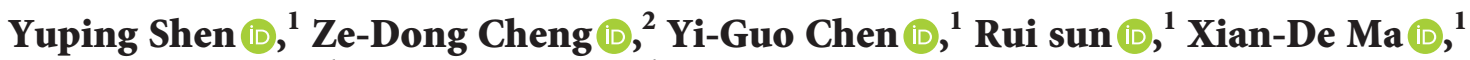 \\ Guo-liang Hou $\mathbb{D}^{1},{ }^{1}$ and Rui Wang $\mathbb{D}^{1}$ \\ ${ }^{1}$ Department of Acupuncture and Moxibustion, Liaoning University of Traditional Chinese Medicine, Shenyang, China \\ ${ }^{2}$ Liaoning University of Traditional Chinese Medicine, \\ Ministry of Education Key Laboratory of Visceral Phenomenon Theory and Application in Traditional Chinese Medicine, \\ Shenyang, China \\ Correspondence should be addressed to Ze-Dong Cheng; 893554622@qq.com and Yi-Guo Chen; cyg05@hotmail.com
}

Received 28 April 2020; Revised 28 September 2020; Accepted 18 October 2020; Published 31 October 2020

Academic Editor: Mingjun Zhu

Copyright (C) 2020 Yuping Shen et al. This is an open access article distributed under the Creative Commons Attribution License, which permits unrestricted use, distribution, and reproduction in any medium, provided the original work is properly cited.

\begin{abstract}
Background and Objective. As a recommended option for 43 diseases by the World Health Organization, electroacupuncture is also being used to treat some others in China. Diet-related intestinal flora imbalance can induce atherosclerosis. This study aims to evaluate how electroacupuncture copes with atherosclerosis through regulating intestinal flora. Methods. In this study, general rabbit conditions, vascular histology, metabolites, and intestinal flora structures were analyzed. Integrated analysis of metabolomics and $16 \mathrm{~S}$ rRNA sequencing were performed. All the rabbits were randomly divided into four groups. The rabbit model of atherosclerosis was established. The histopathological change in the common carotid artery was assessed by HE staining and the structural change in the flora by $16 \mathrm{~S}$ rRNA sequencing. HPLC-TOF-MS and Agilent MPP 12.1 were integrated to identify and screen out differential metabolites. Correlational analyses of every differential metabolite with intestinal flora were integrated on Omicshare platform. Results. Atherosclerotic rabbits showed obvious changes in general conditions, significant fibrous cap and necrotic center on carotid artery, abnormal intestinal bacteria structure, and metabolites levels. Electroacupuncture improved the conditions, reduced lipid deposition on the carotid artery wall, diversified intestinal flora, and normalized host metabolism. Integrated analysis showed that 149 altered metabolites were related to 22 intestinal flora, among which eight intestinal floras and 21 metabolites have relationships with atherosclerosis. Conclusion. Electroacupuncture can effectively reverse atherosclerosis through manipulating the structural feature of intestinal flora to influence the host metabolites. The possible mechanisms involved activating signal pathways through host metabolites or affecting the activity of cardiovascular-related enzymes, or regulating host lipid metabolism directly.
\end{abstract}

\section{Introduction}

Harboring microbes with metabolic functions, intestinal flora is critical to human health. Species spectrum analysis based on 16S rRNA sequencing has comprehensively explored the role of intestinal flora, mainly its structure, in disease development [1]. Besides, metabolomics has been increasingly applied to unravel the functions of human microbiota [2]. Metabolomic analysis focuses on the endogenous metabolites of a biological system, in a holistic view that also appears in Traditional Chinese Medicine (TCM) [3]. Recent years have seen its breakthrough in dealing with cardiovascular disease. These technologies enable us to study on the relationship between intestinal flora and chronic diseases.

In the recent decade, the relationship between intestinal flora and chronic cardiovascular diseases has become a hot topic. The incidence and mortality from atherosclerosis have declined in some countries [4]. It is clear that the intestinal flora can metabolize dietary L-carnitine and choline into trimethylamine, a substance further transformed into trimethylamine-N-oxide (TMAO) in the liver. TMAO can increase cholesterol deposition in vascular cells and accelerate the formation of foam cells and subsequent 
atherosclerosis plaques. However, atherosclerosis is caused by complex factors, and the relationships between metabolites and intestinal flora remain to be discovered. Therefore, in this research, we focused on the intestinal flora associated metabolites that participates in atherosclerosis.

Therapeutic methods for atherosclerosis vary. Acupuncture has emerged as a new clinical option for atherosclerosis in China. Some clinical studies showed that acupuncture can decrease carotid intimal thickness (IMT) and Crouse scores in phlegm dampness constitution carotid atherosclerosis with unique advantages [5]. Another clinical study showed that acupuncture combined with intravenous administration of alprostadil achieve better effect than simple intravenous administration of alprostadil for lower limb atherosclerosis of early diabetes mellitus [6]. In animal experiments, acupuncture has the same effect. A study showed that electroacupuncture at Quchi (LI-11), Zhongwan (CV-12), and Fenglong (ST-4) could regulate HDL-C, LDL-C, and atherosclerosis index [7]. Our previous studies [8] showed that electroacupuncture could reduce the atherosclerotic plaques, regulate the abnormal changes of blood lipid [9], and hemorhelogy in rabbits [10], and point stimulation can optimize the structure of intestinal flora [11]. A study has also found that electroacupuncture can modulate the status of intestinal flora [12]. Intestinal flora is known to have relationship with atheroslcoersis, but the mechanism needs more evidence. Therefore, we designed this research to illustrate how electroacupuncture regulates rabbit intestinal flora to reverse the development of atherosclerosis.

\section{Materials and Methods}

2.1. Animals and Ethical Approval. A total of 24 male New Zealand white rabbits (weighing $2.5 \pm 0.5 \mathrm{~kg}$, aged $2-3$ months) were kept under standardized conditions (light from 6 am to $6 \mathrm{pm}$, temperature $18-20^{\circ} \mathrm{C}$, relative humidity 50-70\%), with free access to food and water. Rabbits were supplied by the Jinan Jinfeng Experimental Animal Company. The certification number is SCXK(Lu)2014-0006. All animal procedures were approved by the Local Ethical Review Panel of the Liaoning University of TCM (Ethical Inspection, No. 21000092017018). After experiment, animals were euthanized with air needle injection.

2.2. Reagents. High-fat diet, consisting of $1 \%$ cholesterol, $3 \%$ lard, $10 \%$ egg yolk powder, and $86 \%$ common ingredients, was processed into pellet feed by Qianmin Animal Feed Processing Center in Yuhong District, Shenyang, China. Other materials included atorvastatin calcium tablets (code: 9H0285991, Beijing Jialin Pharmaceutical Co., Ltd.); penicillin sodium (code: H13020657, North China Pharmaceutical Co., Ltd.); disposable sterile PTCA balloon dilatation catheter $(2.0 \times 15 \mathrm{~mm}$, Beijing Demarc Co., Ltd.); needles (diameter $0.32 \mathrm{~mm}$, length $15 \mathrm{~mm}$, Suzhou Global Co., Ltd.); electroacupuncture apparatus (Suzhou medical products Co., Ltd.); HPLC-grade methanol (Merck, Darmstadt, Germany); distilled water (Mill-Q ultrapure water machine, Millipore, USA); HPLC-grade formic acid (Tianjin Kermel Chemical Company, Tianjin, China). Pipettes, EP tubes, 10\% urethane, heparin, injection bottles, pipette tips, and oil red $\mathrm{O}$ dye were provided by Liaoning University of the TCM laboratory.

2.3. Model Establishment. After one week of adaptive feeding, the rabbits were randomly divided into five groups: control group (normal rabbits, $n=6$ ) and model group (atherosclerotic rabbits, $n=6$ ), AC group (atherosclerotic rabbits + atorvastatin, $n=6$ ), and EA group (atherosclerotic rabbits + electroacupuncture, $n=6$ ). From beginning to the end, the control group was fed with standard diet without any treatment. In model, AC, and EA groups, after four week's high-fat diet ( $120 \mathrm{~g}$ per day), carotid artery balloon injury was made. Then, the high-fat diet was continued for another four weeks.

2.4. Balloon Injury. After $12 \mathrm{~h}$ of fasting and water deprivation, rabbits were anesthetized with $10 \%$ urethane $3 \mathrm{ml} / \mathrm{kg}$ through the ear vein. The skin was cut to expose subcutaneous tissue at midline of neck. The right carotid artery and the right external carotid artery were dissected. After injection of $200 \mu / \mathrm{kg}$ heparin at the ear vein, the right external carotid artery was blocked at distal end. The proximal end was separated, and then a small hole was made at the right external carotid artery. A $3.0 \times 20 \mathrm{~mm}$ PTCA balloon catheter was inserted into the right carotid artery through a point about $4-5 \mathrm{~cm}$ to the right external carotid artery incision. Under 5-6 atmospheres, the saline was used to fill balloon. This process was repeated for three times and each time thirty seconds. The inserted catheter was pulled out, and the right external carotid artery was ligated. Meanwhile, intramuscular injection of 1,000,000 units of penicillin was administered after surgery for three days to prevent infection. After the balloon injury, the control group was fed with normal diet while the others with high-fat diet.

2.5. Experimental Treatment. After model establishment, atorvastatin calcium tablets $(1 \mathrm{mg} / \mathrm{kg} / \mathrm{d})$ were added into high-fat diet in the AC group for four weeks. The EA group was treated with electroacupuncture therapy for four courses.

2.6. Electroacupuncture. Acupoints Neiguan (PC-6), Zusanli (ST-36), and Guanyuan (BL-26) were stimulated with sterile acupuncture needles. The needle was inserted by $0.2-0.3 \mathrm{~cm}$ for PC-6, $0.5-0.8 \mathrm{~cm}$ for ST-36, and $0.3-0.5 \mathrm{~cm}$ for BL-26. PC- 6 was located in the gap of radius and ulna, on the line connecting the rasceta with the ulnar side of the biceps tendon, and $1 / 6$ to the midpoint of the rasceta. ST-36 was located at $2 / 5$ of the line connecting the navel and symphysis pubis. BL-26 was located at the upper $1 / 5$ to the intersection of lateral calf, about $1.2 \mathrm{~cm}$ below the fibular head, $1 \mathrm{~cm}$ behind the posterior tibial crest. According to the rabbit's tolerance and local muscle twitching, the electroacupuncture apparatus was powered with rarefaction wave, $1 \mathrm{~mA}$ 
consuming current, and $2 \mathrm{~Hz}$ frequency. This therapy was performed once a day, 20 minutes per treatment, six days per course, and with an interval of one day after a course.

2.7. Sample Collection. At the end of the treatments, all groups were anesthetized with $10 \%$ urethan $(3 \mathrm{ml} / \mathrm{kg})$ through ear vein. Two milliliters of blood were drawn for metabolomics. After euthanasia, partial fragment of the right carotid artery and fresh feces were collected. The plasma samples and feces were stored in fridge at $-80^{\circ} \mathrm{C}$. Excess tissues of the right carotid arteries were eliminated and fixed with $10 \%$ neutral formalin.

2.8. General Conditions. The general conditions were graded and scored (Table 1). The scores of every rabbits in each group before and after the study were compared $[13,14]$.

\subsection{Histopathology Analysis}

2.9.1. HE Staining. The right carotid artery was embedded by paraffin was transversely sectioned (thickness of $5 \mu \mathrm{m}, 4$ sections per artery) and HE stained. After sealing, the pathological changes in arterial tissue were observed and photographed using a digital microscope.

2.9.2. Red Oil Staining. After $24 \mathrm{~h}$ of fixation, parts of carotid arteries were washed by PBS water for 3 times. The carotid artery was cut longitudinally and immersed in the oil red dye for $1 \mathrm{~h}$. The artery tissue was washed with $75 \%$ ethanol, until the fatty plaque turned red and other parts turned milky white. Then, the artery tissue was washed with distilled water for three times and fixed with needles.

2.10. Plasma Sample Preparation. All plasma samples were unfrozen at room temperature before HPLC-TOF-MS analysis. In HPLC-TOF-MS analysis, every $200 \mu \mathrm{L}$ of plasma was mixed with $600 \mu \mathrm{L}$ of methanol. After vortex-mixing for $2 \mathrm{~min}$, the mixture was centrifuged $\left(7,500 \mathrm{rpm}, 15 \mathrm{~min}, 4^{\circ} \mathrm{C}\right)$ to precipitate the proteins. The supernatant was transited into an EP tube with a proper pipette and dried by nitrogen concentration. The samples were dissolved with $100 \mu \mathrm{L}$ of methanol and centrifuged again $\left(2,000 \mathrm{rpm}, 2 \mathrm{~min}, 4^{\circ} \mathrm{C}\right)$. Finally, $90 \mu \mathrm{L}$ of supernatant was transferred into an autosampler vials with a proper pipette.

2.11. Data Processing and Multivariate Analysis. The mass data were analyzed with Agilent Qualitative Analysis software, Mass Hunter Qualitative analysis software (MHQ, USA) within molecular feather extraction (MFE) and with Agilent MPP software (version 12.1, Agilent Corporation, USA) for alignment and normalization. Using PCA analysis, the metabolites with variable importance in the projection (fold change $>2$ ) and the $P$ values of one-way ANOVA $(P<0.01)$ were selected as potential biomarkers. The potential markers were identified by MPP software with ID Browsers and contrasted in available biochemical databases,
KEGG and National center for biotechnology information (NCBI).

2.12. HPLC-TOF-MS Analysis. The Agilent 1290 HPLC system (Agilent Corporation, USA), Agilent Time of flight Mass Spectrometer equipped with electrospray ionization, and Agilent Poroshell 120 SB-C18 column $(100 \mathrm{~mm} \times 4.6 \mathrm{~mm}$ i.d., $2.7 \mu \mathrm{m}$, Agilent Corp, USA) were used for $t$ HPLC-MS analysis. The column temperature was kept at $35^{\circ} \mathrm{C}$, the flow rate of the mobile phase at $0.4 \mathrm{ml} / \mathrm{min}$, and the injection volume at $4 \mu \mathrm{L}$. Mobile phase consisted of $0.1 \%$ formic acid in water (A) and methanol (B). The column was eluted with $100 \%$ B first, then $20 \% \mathrm{~A}$ and $80 \%$ B for $10.00 \mathrm{~min}$, and $100 \% \mathrm{~B}$ for $20.00 \mathrm{~min}$. The parameters for HPLC-TOF-MS analysis were set as follows: ion source of: ESI, capillary voltage of $3500 \mathrm{~V}$, gas temperature of $250^{\circ} \mathrm{C}$, drying gas of $9 \mathrm{~L} / \mathrm{min}$, nebulizer of $45 \mathrm{psig}$, and fragmentor voltage of $125 \mathrm{~V}$. An injection with a needle of automaticwashing mode was employed on the autosampler.

2.13. Analysis of Intestinal Flora Structure and Multiomics Union. 16S rRNA sequencing was performed by Shanghai Personal Biotechnology Co., Ltd. Multiomics union analysis was performed by Guangzhou Genedenovo Biotechnology Co., Ltd.

\section{Results}

3.1. General Condition Score. During the first week, rabbits were in good condition, and there was no significant difference between groups $(P>0.05)$. However, after modeling, the rabbits gradually showed decreased activity, loss of appetite, diarrhea, dark ear color, and general body lightening. But, the general condition of the rabbits treated with atorvastatin or electroacupuncture was better. The general condition score is shown in Figure 1.

\subsection{Histopathological Results}

3.2.1. HE Staining Results. The aorta tissues (Figure 2) in the control group showed uniform vascular, integrated endothelium, smooth vascular wall without lipid deposition, no foam cell accumulation, and atherosclerotic plaques. In contrast, the tissues in the model group showed obvious fibrous cap and necrotic center, indicating that the atherosclerotic models had been successful established. In AC and EA groups, the thickness of the vascular wall, the level of inflammatory cells, the infiltration of foam cells, and the plaque area decreased after atorvastatin or electroacupuncture.

3.2.2. Red Oil Staining Results. Red oil staining results were showed the changes of atherosclerosis plaque size. After staining, the normal carotid artery tissue in the control group was milky white, while in the model group the lipid deposited on the endothelium, showing orange red (Figure 3). The orange red areas in the AC and EA groups were smaller than those in the model group. 
TABLE 1: Scores of conditions.

\begin{tabular}{lccccc}
\hline Score & Activity & Auricle color & Surplus food $(\mathrm{g})$ & Weight & Stool consistency \\
\hline 1 & Active move, lively & Ruddy & $<60$ & Gained $>0.8 \mathrm{~kg}$ & Round and smooth \\
2 & Active move, not lively & Pale & $60-100$ & Gained $<0.8 \mathrm{~kg}$ & Diarrhea, amount less than $10 \mathrm{ml}$ \\
3 & Inactive & Gloomy & $>100$ & Lose weight & Diarrhea, amount more than $10 \mathrm{ml}$ \\
\hline
\end{tabular}

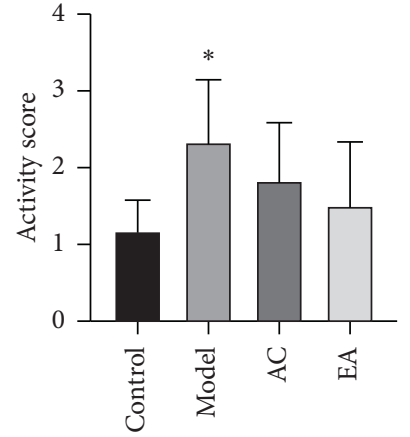

(a)

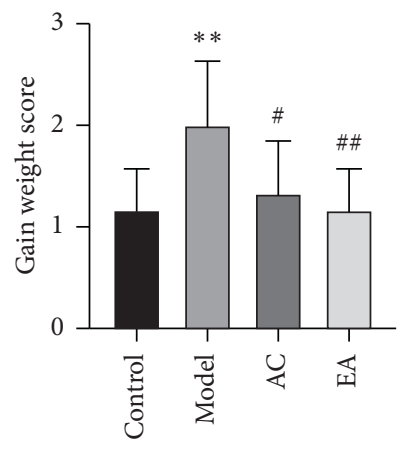

(d)

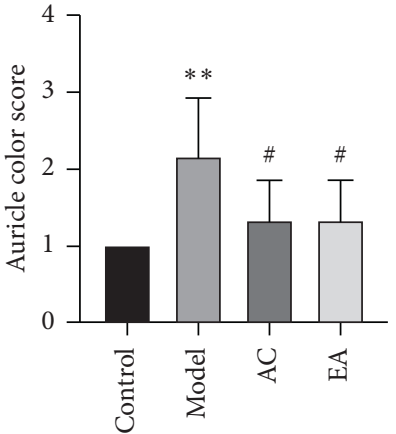

(b)

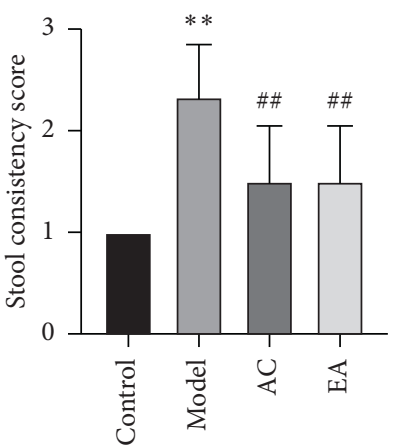

(e)

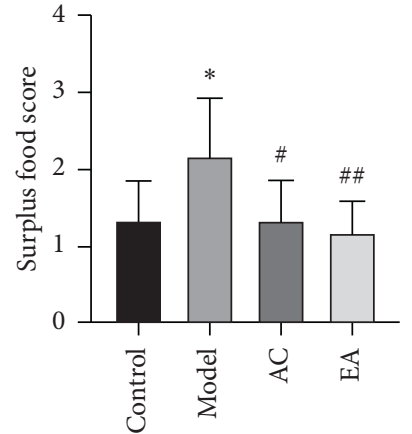

(c)

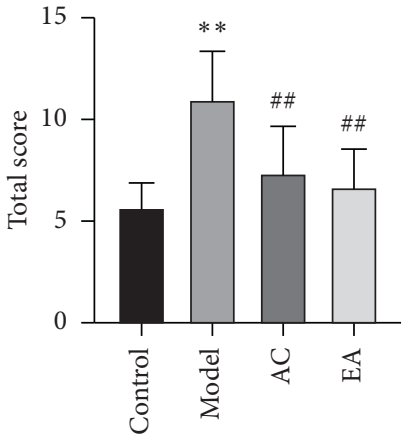

(f)

Figure 1: General condition scores. ${ }^{*} P<0.05$ vs. control, ${ }^{* *} P<0.01$ vs. control. ${ }^{\#} P<0.05$ vs. model, and ${ }^{\# \#} P<0.01$ vs. model.

3.3. Intestinal Flora Structure. The top ten most abundant of intestinal flora were visualized. Different color means different flora, and the length of each color column means the abundance of corresponding flora. The results showed that, on family level, the abundances of intestinal flora in different groups were different (Figure 4). ACE and Simpson indexes were used to show abundance and diversity (Table 2).

3.4. Metabolic Analysis Results. Low-molecular weight metabolites were separated completely in $20 \mathrm{~min}$. The results of ANOVA using MPP software and principal component analysis (PCA) are shown in Figure 5. Obviously, the metabolites in different groups showed differences. The dimensional patterns in AC and EA groups were closer to that in the control group, which meant the metabolites in these three groups are similar. Actually, 222 metabolites showed statistic difference. Compared with control group, there were 70 metabolites upregulated and 151 metabolites downregulated. Compared with the model group, 117 metabolites in AC group and 84 metabolites in EA group were upregulated, while 86 metabolites in AC group and 136 metabolites in EA group were downregulated. The 132 metabolites in AC group and 129 metabolites in EA group showed the same tendency with control group, which indicated that electroacupuncture not only regulated the structure of intestinal flora but also the production of host metabolites.

\subsection{Integration of Metabolomics and $16 S$ rRNA Sequencing.} The results of O2PLS analysis are shown in the Figure 6(a). The dots represent bacterium or metabolites. The greater the absolute value in the coordinates, the greater the degree of association between this element and another omics. Among the 222 metabolites with differential levels, 149 metabolites showed relationships with 22 intestinal flora $(P<0.05)$ (Figure 6(b)). According to the Method for diagnosing heart disease through bacterial metagenomic analysis [15], the changes of Thermogemmatisporaceae, Flavobacteriaceae, Verrucomicrobiaceae, S24-7, Lachnopiraceae, Bifidobacteriaceae, Turicibacteraceae, Veillonellaceae, Ruminococcaceae, and Clostridiales could be used in the diagnosis of cardiovascular disease, including atherosclerosis. These 10 families of intestinal bacterium were definitely related to 45 metabolites. According to KEGG, Lipid MAP, METLIN, and HMDB databases, 21 metabolites have been confirmed to be associated with the development of atherosclerosis. In this study, electroacupuncture regulated the 8 intestinal bacteria 


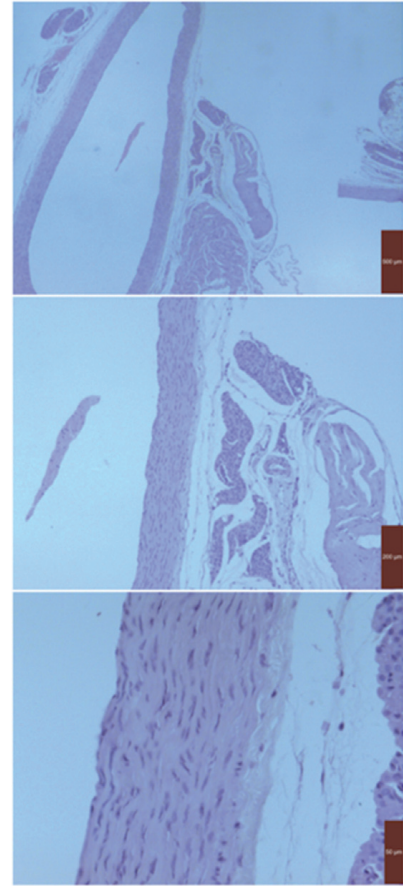

(a)

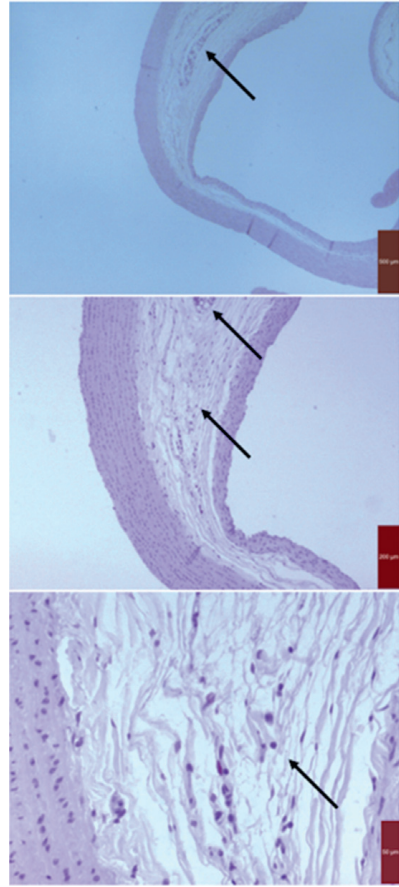

(b)
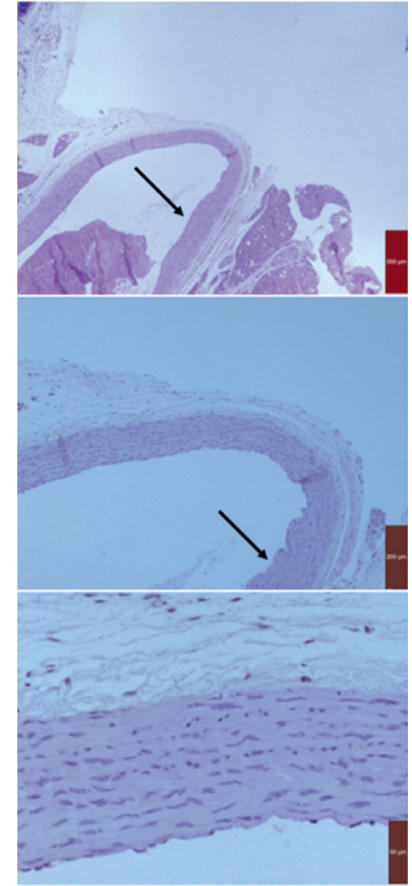

(c)

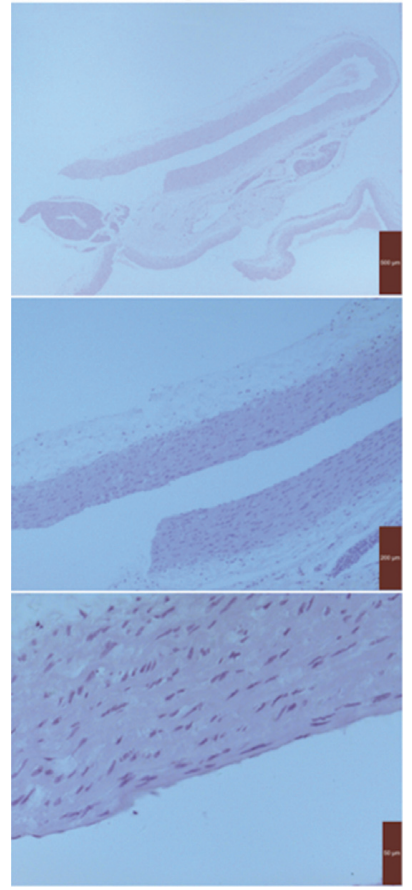

(d)

Figure 2: HE staining of atherosclerotic plaques $(\times 40, \times 100$, and $\times 400)$. (a) Control group. (b) Model group. (c) AC group. (d) EA group.

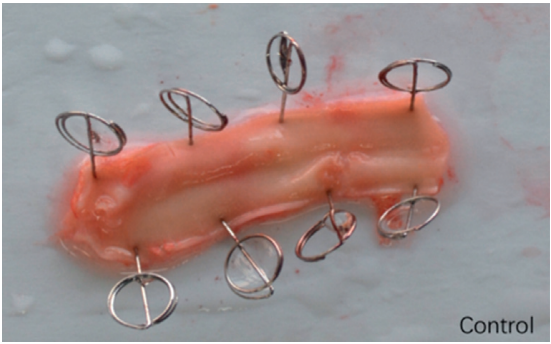

(a)

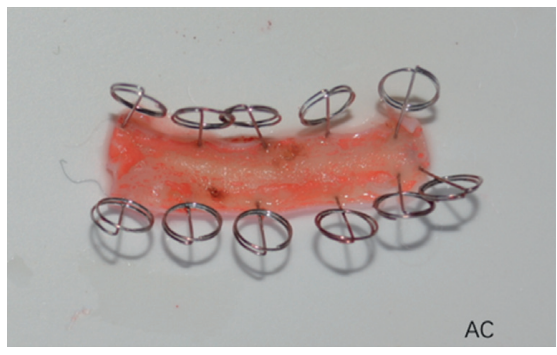

(c)

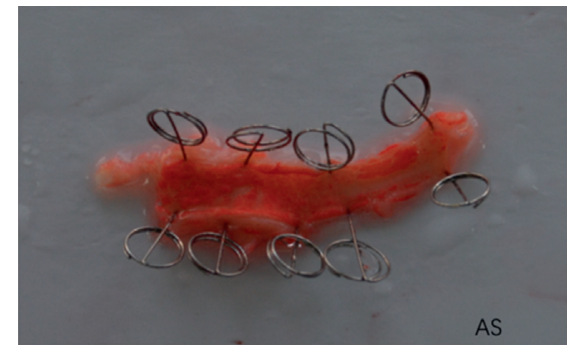

(b)

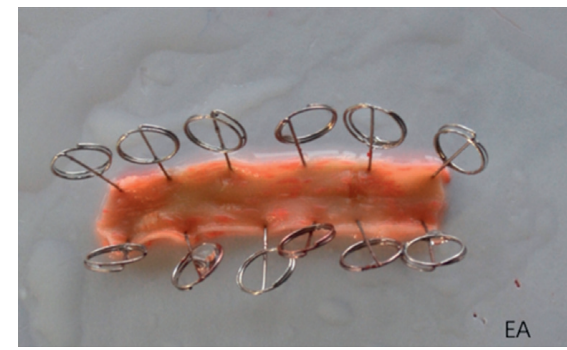

(d)

Figure 3: Oil red O staining of atherosclerotic plaque size.

TAble 2: Alpha index of intestinal flora. ${ }^{*} P<0.05$ vs. control, ${ }^{* *} P<0.01$ vs. control, and ${ }^{\# \#} P<0.05$ vs. model.

\begin{tabular}{lcr}
\hline Group & ACE & Simpson \\
\hline Control & $1466.1383 \pm 165.9871$ & $0.9826 \pm 0.0066$ \\
Model & $1050.4783 \pm 141.9394^{*}$ & $0.9548 \pm 0.0276^{* *}$ \\
AC & $1305.4950 \pm 134.5323^{\#}$ & $0.9789 \pm 0.0042^{\#}$ \\
EA & $1308.0183 \pm 75.8283^{\#}$ & $0.9768 \pm 0.0082^{\#}$ \\
\hline
\end{tabular}




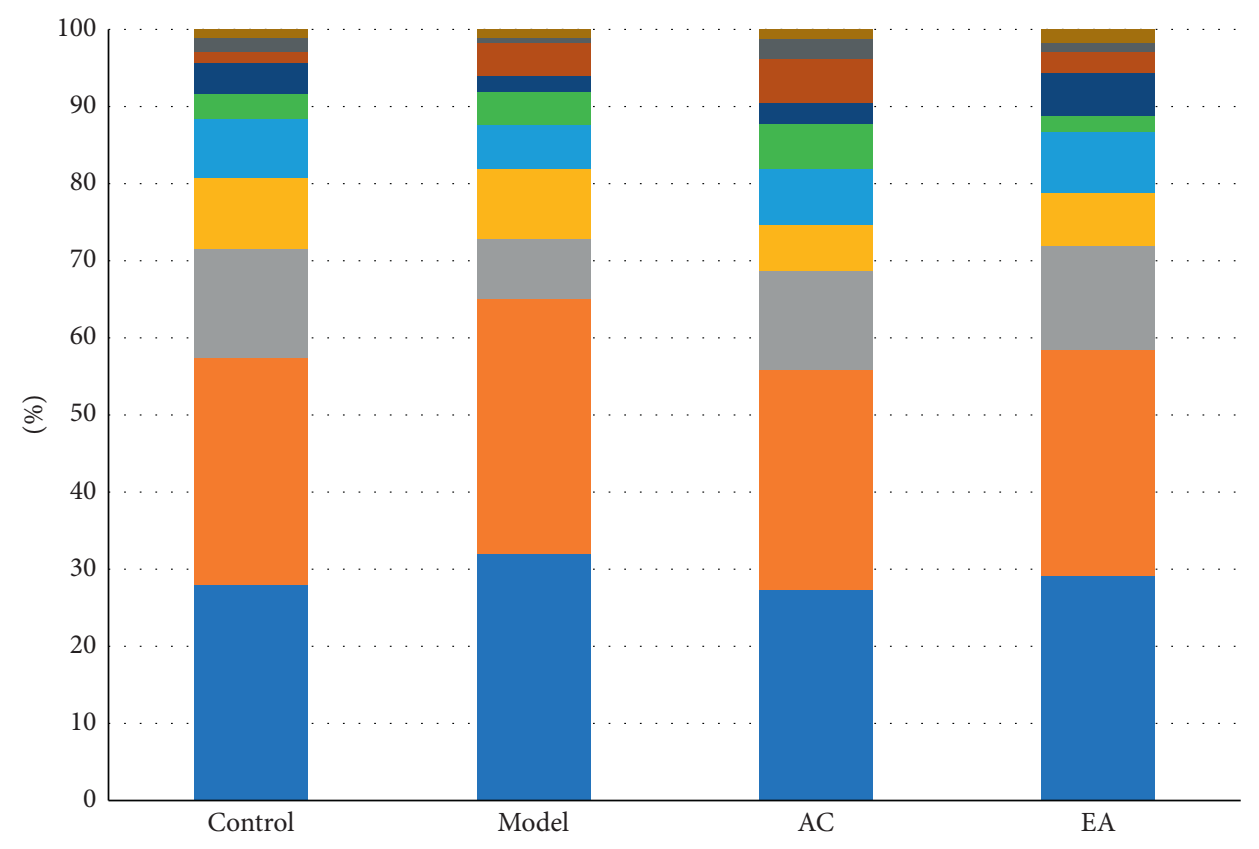

FIGURE 4: Intestinal flora abundance on family level.

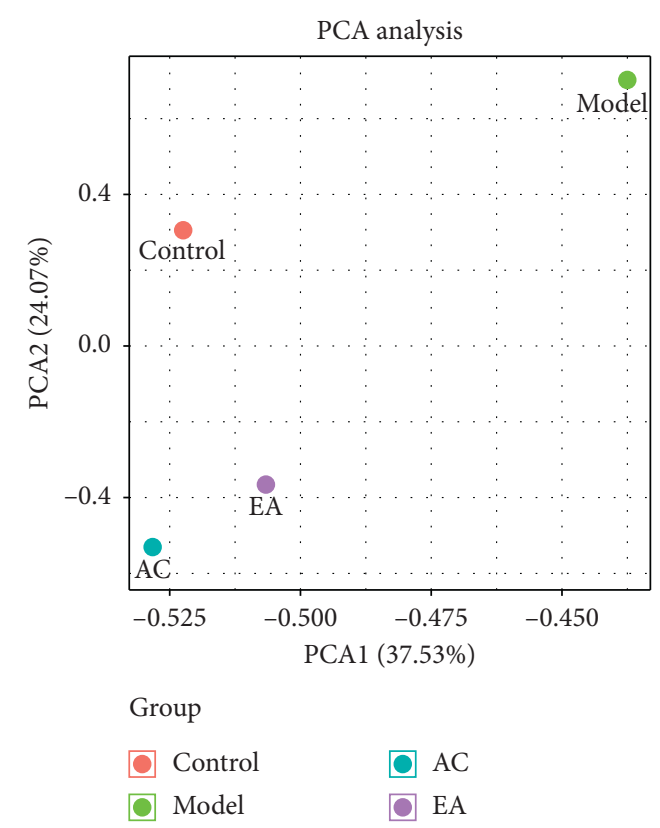

FIgURE 5: PCA of metabolites in different groups.

to influence 21 host metabolites to reverse atherosclerosis (Figure 6(c)).

\section{Discussion}

TCM holds that atherosclerosis develops with the stagnant $q i$ and blood, usually caused by vascular phlegm and stasis. At present, the mechanism of atherosclerosis is not fully understood, but it is certain that the occurrence and development of atherosclerosis are closely related to the abnormal metabolic function of organism. The intestinal flora parasitizes in the intestines of animals and participates in the metabolic processes of the host. Intestinal flora has become a new therapeutic target for various metabolic diseases [16].

In previous study, three relevant findings that can support the use of electroacupuncture as an intervention to treat atherosclerosis. Firstly, point stimulation could change the structural features of intestinal flora [11]. Secondly, electroacupuncture has a good regulatory effect on myocardial ischemia diseases [17]. Thirdly, electroacupuncture could increase the concentration of drugs in specific viscera, which means electroacupuncture combined with drugs will achieve synergistic effect and improve drug efficacy. Besides, it has shown that stimulating PC- 6 can benefit the coronary blood flow [18], stimulating ST-36 can decrease blood lipid [19] and stimulating BL-36 can optimize the structure of intestinal flora [20]. This is the mainly reason that why we choose electroacupuncture as the intervention in this study.

4.1. General Condition and TCM. According TCM theory, atherosclerosis is caused by the combination of phlegm, blood stasis, and toxin. In human body, atherosclerosis will develop with some signs and symptoms, like chest tightness, asthma, fatigue, obesity, or emaciation. In rabbits, fur, ear color, activity, weight gain, and feces condition are important indices, for example, the auricle color could be related with blood stasis. In this study, those symptoms worsen after modeling and relieve after treatment.

4.2. Changes in Histopathology. In this study, electroacupuncture reduced atherosclerosis plaque in EA groups. Favorable morphological changes confirmed the antiatherosclerotic effect of electroacupuncture. This incidence verified the antiatherosclerotic effect of electroacupuncture for atherosclerosis qualitatively. 

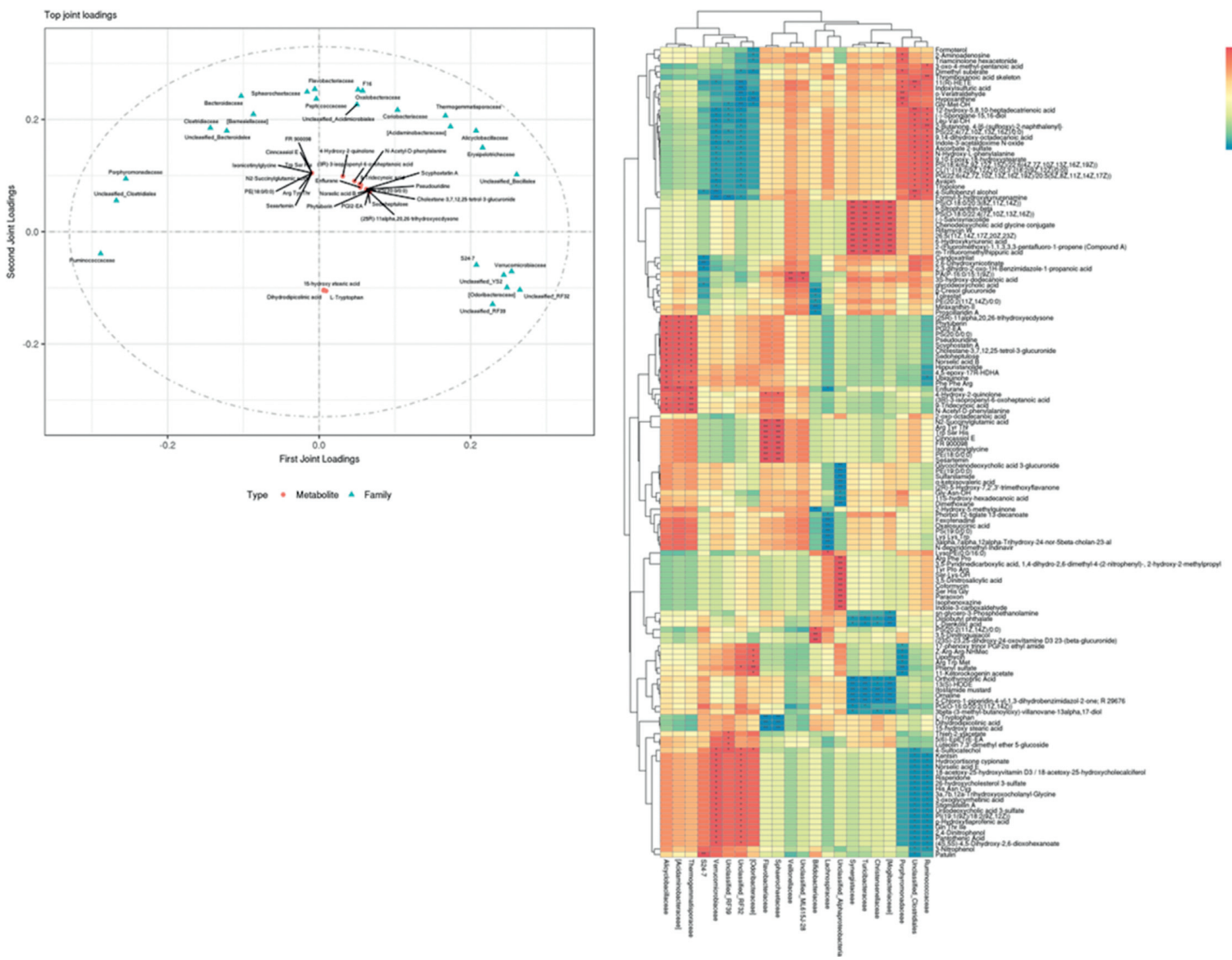

(a)

(b)

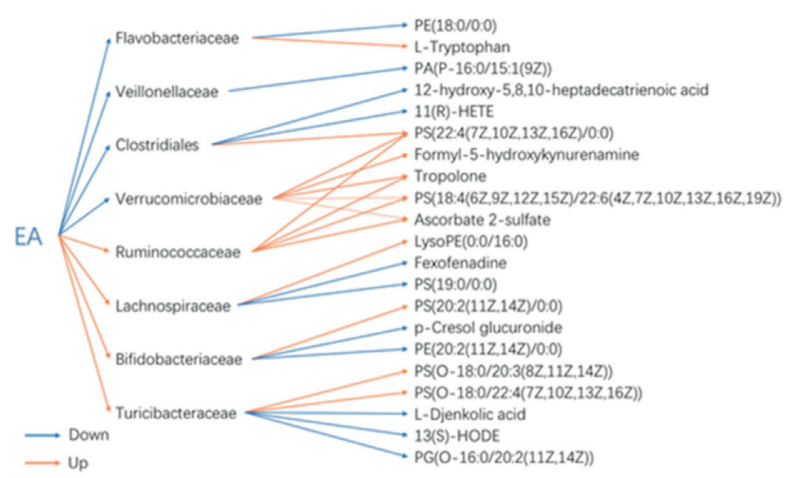

(c)

Figure 6: (a) Integrated metabolomics and $16 \mathrm{~S}$ rRNA sequencing. ${ }^{*} P<0.05$; ${ }^{* *} P<0.01$. (b) O2PLS analysis. (c) Possible methods.

4.3. Structural Feature Changes in Intestinal Flora by Electroacupuncture. Normal intestinal flora maintains homeostasis. Drug may decrease the probiotics, enrich the pathogenic bacteria or opportunistic bacterium [21], and overturn the structural features of intestinal flora [22]. In this study, the differences of the structural features of intestinal flora were obvious, suggesting that the occurrence of atherosclerosis may be related to abnormal structural features of the intestinal flora, and electroacupuncture can regulate this state.
4.4. Metabolites in Plasma Were Different between Groups. In the present study, $16 \mathrm{~S}$ rRNA sequencing showed that either of atherosclerosis, atorvastatin application, and electroacupuncture could change intestinal flora structural features. In order to determine whether this change has an impact on the host, this study selected nontargeted metabonomics to test plasma metabolites. Our results showed that either of atherosclerosis lesions, atorvastatin application, and electroacupuncture could change plasma metabolism levels among the groups. The 
PCA patterns in model and control groups were different, which indicated that atherosclerosis had bad effect on the metabolism of the body. Interestingly, the PCA pattern in the EA group was different to that in model group, but similar to that in the control group, which demonstrates that electroacupuncture could have some therapeutic effect on this metabolic abnormality. And, some of 222 differentially produced metabolites were directly related to atherosclerosis: Ascorbate 2sulfate [23, 24], hypoxanthine [25], Hippurate [26], 13(S)-HODE [27], inositol [28], 6-keto-PGF1 $\alpha$ [29], Nacetylneuraminic acid [30], ubiquinone [31], glucuronide [32], L-tryptophan [33], 15(S)-HETE [34], etc. However, the current evidence is still unable to confirm whether these metabolic changes were caused by intestinal flora or not, so it was necessary to perform integrated analysis of two omics.

4.5. Almost 67\% Differential Metabolites Were Related to Intestinal Flora. The integrated analysis of omics showed that $67 \%$ of 222 differential plasma metabolites were closely related to 22 intestinal flora, suggesting that intestinal flora is not only an important participant in the development of atherosclerosis but also a medium for electroacupuncture to treat atherosclerosis.

The host metabolites can be influenced by electroacupuncture through intestinal flora to activate some signal pathway. Taking Turicibacteriaceae as an example, electroacupuncture can increase its abundance in atherosclerotic rabbits, decrease the metabolites 13(S)-HODE and L-Djenkolic acid in plasma, and then eliminate the atherosclerotic plaque. L-djenkolic acid [35] could produce oxygen ion to trigger low-density lipoprotein oxidation reaction, which will accelerate the formation of atherosclerotic plaque. 13(S)-HODE is a kind of trans-unsaturated fatty acid, which is harmful to the cardiovascular system; however, the fact is that 13 (S)-HODE is negatively correlated with Turicibacteriaceae, but its level in the plasma is high. The reason may be that one metabolite may be affected by multiple intestinal flora. 13(S)-HODE is also affected by Christensenellaceae and (Mogibacteriaceae). 13(S)-HODE is a ligand in the PPAR signal pathway existing in adipocytes. Peroxisome proliferator-activated receptors (PPARs) are nuclear hormone receptors that can be activated by fatty acids and their derivatives, including three major members: $\operatorname{PPAR} \alpha, \operatorname{PPAR} \beta / \delta$, and $\operatorname{PPAR} \gamma$. A lot of evidence showed that atorvastatin lowers lipid level through activating PPARs. This is why 13(S)-HODE is higher after electroacupuncture. Moreover, we can speculate that electroacupuncture may also relieve atherosclerosis by employing the intestinal flora to regulate the levels of host metabolites and then activate PPARs.

Host metabolites can be affected by electroacupuncture through intestinal flora to adjust the activity of cardiovascularrelated enzymes, Tropolone, for instance, a kind of catechol-Omethyltransferase (COMT) inhibitor. COMT has been implicated in both depression and cardiovascular disease (CVD). A study showed that high COMT activity genotype Val158Met, associated with activity of COMT, can increase the risk of
CVD [36]. Moreover, Hinokitiol, a derivative of Tropolone, can mediate $\mathrm{p}$-JNK and $\mathrm{p}$-PLC $\gamma 1$ signaling pathways to achieve antiatherosclerotic plaque effects [37]. In this study, electroacupuncture can upregulate Ruminococcaceae and downregulate Verrucomicrobiaceae to upregulate Tropolone in plasma, which indicated that electroacupuncture may inhibit the activity of COMT by upregulating Tropolone through intestinal flora to prevent CVD.

Electroacupuncture also can influence host lipids through intestinal flora to adjust lipid metabolism, such as PS(22:4(7Z, 10Z, 13Z, 16Z)/0:0), LysoPE(0:0/16:0), PA(P-16:0/15:1(9Z)), PS(O-18:0/20:3(8Z, 11Z, 14Z)), PS(O-18:0/22:4(7Z, 10Z, 13Z, 16Z)), and PG(O-16:0/20: $2(11 Z, 14 Z))$. They belong to glycerophospholipid category, and electroacupuncture can adjust them through intestinal flora.

This study has some limitations. Firstly, the range of the mass-to-charge ratio that can be predicted by HPLC/ESITOF-MS is 100 to 1500 , so beyond this range, molecular information cannot be captured. Secondly, the metabolomics in this study is nontargeted and metabolites cannot be accurately quantified. Therefore, the results of this study need to be verified by more experiments.

\section{Conclusion}

Electroacupuncture can effectively reverse atherosclerosis through manipulating the structural feature of intestinal flora to influence the host metabolites. The possible mechanisms involved activating the signal pathways through metabolites, or affecting the activity of cardiovascular-related enzymes, or regulating host lipid metabolism directly.

\section{Data Availability}

The data used to support the article are available in the article.

\section{Disclosure}

Zedong-Cheng is the co-first author.

\section{Conflicts of Interest}

The authors declared no potential conflicts of interest with respect to the research, authorship, and/or publication of this article.

\section{Authors' Contributions}

YC and ZC designed the experiment. XM, RW, and RS performed the animal experiment and histopathology analysis and biochemical tests. YS performed metabolomics analysis and paper writing. GH and YS reviewed and edited the manuscript. 


\section{Acknowledgments}

The authors are very grateful to Prof. Xiansheng-Meng for assistance with the metabolomics. They are grateful to Shanghai Personal Biotechnology Co., Ltd, for assisting in 16S rRNA sequencing and grateful to Guangzhou Gene Denovo Biotechnology Co., Ltd, for assisting in integrated analysis. This paper is from the National Natural Science Foundation of China (No.81674078) and the Open Fund of Ministry of Education Key Laboratory of Visceral Phenomenon Theory and Application of Liaoning University of Traditional Chinese Medicine (zyzx1708).

\section{Supplementary Materials}

All data that involved in this manuscript have been uploaded. (Supplementary Materials)

\section{References}

[1] Y. K. Chan, M. S. Brar, P. V. Kirjavainen et al., "High fat diet induced atherosclerosis is accompanied with low colonic bacterial diversity and altered abundances that correlates with plaque size, plasma A-FABP and cholesterol: a pilot study of high fat diet and its intervention with Lactobacillus rhamnosus GG (LGG) or telmisartan in $\mathrm{ApoE}^{-/-}$mice," BMC Microbiology, vol. 16, no. 1, p. 264, 2016.

[2] J. Chong and J. Xia, "Computational approaches for integrative analysis of the metabolome and microbiome," $\mathrm{Me}$ tabolites, vol. 7, no. 4, p. 62, 2017.

[3] M. X. Chen, S.-Y. Wang, C.-H. Kuo, and I.-L. Tsai, "Metabolome analysis for investigating host-gut microbiota interactions," Journal of the Formosan Medical Association, vol. 118, no. 1, pp. S10-S22, 2019.

[4] W. Herrington, B. Lacey, P. Sherliker, J. Armitage, and S. Lewington, "Epidemiology of atherosclerosis and the potential to reduce the global burden of atherothrombotic disease," Circulation Research, vol. 118, no. 4, pp. 535-546, 2016.

[5] H. Liang, L. Meng, Y. Yuan et al., "Clinical observation on the treatment of carotid atherosclerosis in phlegm dampness constitution," Guangdong Medical Journal, vol. 39, no. 13, pp. 2070-2073, 2018.

[6] P. Liang, A. Wei, and Z. Gu, "Effects of acupuncture at stellate ganglion on lower limb atherosclerosis of early diabetes mellitus," Zhongguo Zhen Jiu, vol. 36, no. 5, pp. 476-480, 2016.

[7] G. X. Zhang, J. L. Miao, Z. Y. Zhang, H. J. Wang, and L. X. Ji, "Regulation effects of electroacupuncture with different acupoint combinations on blood lipid in rats with hyperlipemia," Zhongguo Zhen Jiu, vol. 34, no. 9, pp. 894-897, 2014.

[8] Z. Cheng, Y. Ning, and R. Wang, "Effects of electroacupuncture on expression of CD36 in peritoneal macrophages of rabbits with atherosclerosis," Zhongguo Zhen Jiu, vol. 38, no. 2, pp. 179-184, 2018.

[9] H. Luo and Z. Cheng, "Effects of Electroacupuncture on blood lipid and hemorheology in atherosclerotic rabbits," Liaoning Journal of Traditional Chinese Medicine, vol. 45, no. 8, pp. 1761-1790, 2018.

[10] S. H. Zhang and Z. D. Cheng, "Effects of electroacupuncture on CYP7A1 expression in liver of rabbits with atherosclerosis," Zhongguo Zhen Jiu, vol. 39, no. 1, pp. 59-64, 2019.

[11] S. Wang, Z. Cheng, D. Jin, and Y. Chen, "Moxibustion at Front-Mu point of abdomen for intestinal dysbacteriosis in rats," Journal of Acupuncture and Tuina Science, vol. 9, no. 1, pp. 21-25, 2011.

[12] J. Xu, X. Zheng, K.-K. Cheng et al., "NMR-based metabolomics reveals alterations of electro-acupuncture stimulations on chronic atrophic gastritis rats," Scientific Reports, vol. 7, no. 1, Article ID 45580, 2017.

[13] G. C. Mcmillan, J. H. Whiteside, and G. L. Duff, "The effect of undernutrition on cholesterol atherosclerosis in the rabbit," Journal of Experimental Medicine, vol. 99, no. 3, pp. 261-274, 1954.

[14] D. C. Cavallini, R. Bedani, L. Q. Bomdespacho, R. C. Vendramini, and E. A. Rossi, "Effects of probiotic bacteria, isoflavones and simvastatin on lipid profile and atherosclerosis in cholesterol-fed rabbits: a randomized double-blind study," Lipids Health Disease, vol. 8, no. 1, 2009.

[15] Y. Jin, Method for Diagnosing Heart Disease through Bacterial Metagenomic Analysis, https://kns.cnki.net/kcms/detail/detail. aspx?FileName=CN110392741A\&DbName=SCPD2019, 2019.

[16] Y. Qi, S. Kim, E. M. Richards, M. K. Raizada, and C. J. Pepine, "Gut microbiota: potential for a unifying hypothesis for prevention and treatment of hypertension," Circulation Research, vol. 120, no. 11, pp. 1724-1726, 2017.

[17] S. Wang, L. Ren, L. Jia et al., "Effect of acupuncture at Neiguan (PC 6) on cardiac function using echocardiography in myocardial ischemia rats induced by isoproterenol," Journal of Traditional Chinese Medicine, vol. 35, no. 6, pp. 653-658, 2015.

[18] P. Li, K. F. Pitsillides, S. V. Rendig, H.-L. Pan, and J. C. Longhurst, "Reversal of reflex-induced myocardial ischemia by median nerve stimulation: a feline model of electroacupuncture," Circulation, vol. 97, no. 12, pp. 1186-1194, 1998.

[19] E. L. W. Santos, B. H. M. Dias, A. C. R. d. Andrade et al., "Effects of acupuncture and electroacupuncture on estradiolinduced inflammation and oxidative stress in health rodents," Acta Cirurgica Brasileira, vol. 28, no. 8, pp. 582-588, 2013.

[20] Z. Cheng, Y. Chen, and T. Zhang, "Effect of mild moxibustion on intestinal flora of chronic fatigue rats," Chinese Journal Infarction Traditinal Chinese Medicine, vol. 20, no. 1, pp. 45-63, 2013.

[21] M. Y. Yoon and S. S. Yoon, "Disruption of the gut ecosystem by antibiotics," Yonsei Medical Journal, vol. 59, no. 1, pp. 4-12, 2018.

[22] G. A. Weiss and T. Hennet, "Mechanisms and consequences of intestinal dysbiosis," Cellular and Molecular Life Sciences, vol. 74, no. 16, pp. 2959-2977, 2017.

[23] E. Hayashi, J. Yamada, M. Kunitomo, M. Terada, and M. Sato, "Fundamental studies on physiological and pharmacological actions of L-ascorbate 2-sulfate. V. On the hypolipidemic and antiatherosclerotic effects of L-ascorbate 2-sulfate in rabbits," The Japanese Journal of Pharmacology, vol. 28, no. 1, pp. 61-72, 1978.

[24] F. J. Finamore, R. P. Feldman, and G. E. Cosgrove, "L-ascorbic acid, L-ascorbate 2-sulfate, and atherogenesis," International Journal for Vitamin and Nutrition Research, vol. 46, no. 3, pp. 275-285, 1976.

[25] H. M. Ryu, Y. J. Kim, E. J. Oh et al., "Hypoxanthine induces cholesterol accumulation and incites atherosclerosis in apolipoprotein E-deficient mice and cells," Journal of Cellular and Molecular Medicine, vol. 20, no. 11, pp. 2160-2172, 2016.

[26] M. Huang, R. Wei, Y. Wang, T. Su, P. Li, and X. Chen, "The uremic toxin hippurate promotes endothelial dysfunction via the activation of Drp1-mediated mitochondrial fission," Redox Biology, vol. 16, pp. 303-313, 2018. 
[27] K. Zha, C. Zuo, A. Wang et al., "LDL in patients with subclinical hypothyroidism shows increased lipid peroxidation," Lipids in Health and Disease, vol. 14, no. 1, p. 95, 2015.

[28] I. De Meyer, W. Martinet, C. E. Van Hove et al., "Inhibition of inositol monophosphatase by lithium chloride induces selective macrophage apoptosis in atherosclerotic plaques," British Journal of Pharmacology, vol. 162, no. 6, pp. 1410-1423, 2011.

[29] J. Wang, L. Wang, H. Yang et al., "Prevention of atherosclerosis by Yindan Xinnaotong capsule combined with swimming in rats," BMC Complementary and Alternative Medicine, vol. 15, no. 1, p. 109, 2015.

[30] P. Hou, S. Hu, J. Wang et al., "Exogenous supplement of $\mathrm{N}$-acetylneuraminic acid improves macrophage reverse cholesterol transport in apolipoprotein E-deficient mice," Lipids in Health and Disease, vol. 18, no. 1, p. 24, 2019.

[31] I. Zeb, N. Ahmadi, J. Kadakia et al., "Aged garlic extract and coenzyme Q10 have favorable effect on inflammatory markers and coronary atherosclerosis progression: a randomized clinical trial," Journal of Cardiovascular Disease Research, vol. 3, no. 3, pp. 185-190, 2012.

[32] Y. Kawai, " $\beta$-Glucuronidase activity and mitochondrial dysfunction: the sites where flavonoid glucuronides act as anti-inflammatory agents," Journal of Clinical Biochemistry and Nutrition, vol. 54, no. 3, pp. 145-150, 2014.

[33] M. J. Forteza, K. A. Polyzos, R. Baumgartner et al., "Activation of the regulatory T-cell/indoleamine 2,3-dioxygenase Axis reduces vascular inflammation and atherosclerosis in hyperlipidemic mice," Frontiers in Immunology, vol. 9, p. 950, 2018.

[34] V. Kundumani-Sridharan, E. Dyukova, D. E. Hansen, and G. N. Rao, "12/15-Lipoxygenase mediates high-fat diet-induced endothelial tight junction disruption and monocyte transmigration: a new role for 15(S)-hydroxyeicosatetraenoic acid in endothelial cell dysfunction," Journal of Biological Chemistry, vol. 288, no. 22, pp. 15830-15842, 2013.

[35] J. W. Heinecke, H. Rosen, L. A. Suzuki, and A. Chait, "The role of sulfur-containing amino acids in superoxide production and modification of low density lipoprotein by arterial smooth muscle cells," The Journal of Biological Chemistry, vol. 262, no. 21, pp. 10098-10103, 1987.

[36] A. Almas, Y. Forsell, V. Millischer, J. Möller, and C. Lavebratt, "Association of Catechol-O-methyltransferase (COMT Val158Met) with future risk of cardiovascular disease in depressed individuals-a Swedish population-based cohort study," BMC Medical Genetics, vol. 19, no. 1, p. 126, 2018.

[37] P.-S. Yang, M.-J. Wang, T. Jayakumar et al., "Antiproliferative activity of Hinokitiol, a Tropolone derivative, is mediated via the inductions of p-JNK and p-plc $\gamma 1$ signaling in PDGF-BBstimulated vascular smooth muscle cells," Molecules, vol. 20, no. 5, pp. 8198-8212, 2015. 